\title{
New Improved Unsharp Masking Methods Compatible with Ultrasound B-Mode Imaging
}

\author{
Asraf Mohamed Moubark, Thomas M. Carpenter, David M. J Cowell, Sevan Harput and Steven Freear \\ Ultrasound Group, School of Electronic and Electrical Engineering, University of Leeds, UK. \\ E-mail: elamm@leeds.ac.uk and S.Freear@leeds.ac.uk
}

\begin{abstract}
In medical ultrasound B-mode imaging, by increasing the image contrast, spatial and temporal resolution, it will help to improve the diagnostic and decision making process. Previously the unsharp masking (UM) techniques have been successfully implemented for digital image processing as edge enhancement techniques. However, the outcomes of the method are limited and influenced by the image formats conversion and lower dynamic range. Moreover the conventional UM technique sensitive to noise due to the presence of linear high pass filter which cannot discriminate signal from noise. Furthermore, the technique only enhances the image in darker compared to lighter regions. To overcome those issues, a new improved UM method compatible with ultrasound B-mode imaging was applied to compound plane wave imaging (CPWI). The received radio frequency (RF) signal was beamformed with delay and sum (DAS) and filtered delay and sum (FDMAS). Results show that the proposed techniques are not only able to improve the image contrast but also the spatial resolution. The UM technique manages to improve the B-mode image lateral resolution (LR) by $18.36 \%$ and $10.25 \%$ as well as reduced the peak side lobes (PSL) by 16.67 dB and 21.24 dB on DAS and FDMAS respectively when compounded with CPWI, $N=13$. For the same number of compounding and beamforming order, the image contrast ratio (CR) has been improved by $10.35 \mathrm{~dB}$ and $7.39 \mathrm{~dB}$ accordingly.
\end{abstract}

\section{INTRODUCTION}

Ultrasound B-mode images are prone to clutter noise that often obscures anechoic regions such as cysts, carotid artery and blood vessels. Clutter noise sources are from reverberation, off-axis side lobes, phase abbreviations, edge waves and random acoustic noise [1]. Clutter noise can be reduced by using filtering, beamforming and post processing techniques such as contrast enhanced delay and sum (CEDAS), dual apodization with cross correlation (DAX), minimum variance (MV) and compound plane wave imaging (CPWI) [1]-[4]. All the proposed methods come with some limitation. CEDAS and DAX only manage to reduce the clutter noise without improving the image spatial resolution. The MV involves high computational complexity. While CPWI with DAS were only able to reduce the clutter noise to a certain extent at the cost of reducing the temporal resolution. Thus to overcome those constraints, a filtering technique known as unsharp masking (UM) which has been applied in digital image processing has been explored in this work. The main objective of this work is to reduce the clutter noise and improve the spatial resolution in ultrasound B-mode image.

\section{Materials And Methods}

\section{A. Unsharp Masking}

The UM is a post processing technique used to improve the digital image details by enhancing the high frequency parts [5]. Conventional UM can be represented by the follwoing equation :

$$
u_{\text {con }}[a, b]=h[a, b]+\lambda|h[a, b]-k * h[a, b]|
$$

where $u_{\text {con }}[a, b]$ is unsharp masked image, $h[a, b]$ is the original digital image, $k$ is blurring kernel and $\lambda$ is weightage or positive scaling factor that controls the level of high frequency components added to the original image. In conventional UM the application of linear high pass filter makes the techniques sensitive to noises. This results undesirable distortion even in slightly noisy regions. Also a unique weightage applied as a scale for the high frequency component produced unpleasant overshoot artifact. Furthermore, the UM techniques enhances more the images on high compared to low and medium contrast regions. Thus in this work the conventional UM technique has been modified to suite ultrasound B-mode imaging principle. The new proposed UM is an iterative scheme that computes successive improved images as given by:

$$
f_{j}(x, z)= \begin{cases}f_{j-1}+\lambda_{1}\left(f_{j-1}-f_{j-1}^{*}\right), & f_{j-1}-f_{j-1}^{*} \geq 0 \\ f_{j-1}+\lambda_{2}\left(f_{j-1}-f_{j-1}^{*}\right), & f_{j-1}-f_{j-1}^{*}<0\end{cases}
$$

where $j$ is number of iteration cycles, $f_{j}(x, z)$ is unsharp masked image, $f_{j-1}^{*}$ is non-coherent CPWI, $\lambda_{1}$ and $\lambda_{2}$ represents two different weigthages scaling factor for positive and negative errors.

The UM process starts with subtracting the coherent CPWI signal, $f_{0}$ with non-coherent CPWI signal, $f_{0}^{*}$ before the log compression process takes place. The difference or errors produced then multiplied with different weightages according to their polarity. Through many experiments trials, $\lambda_{1}$ and $\lambda_{2}$ have been assigned with 0.77 and 0.07 . The reason behind this selection is to control the attenuation level of the clutter noise. Applying same weightages for both positive and negative errors will lead to high dynamic range and turns the grey parts 
on the speckle to black regions. The scaled errors added back to the coherent CPWI, $f_{0}$ signals and the whole steps repeated for three iteration. Beyond three iterations, the quality of the $\mathrm{B}$-mode image worsen with very low level of CNR.

\section{B. Filtered Delay Multiply and Sum Beamforming}

The initial process in FDMAS is the same as DAS. Here, the signal $s_{i}(t)$ is the RF signal received by each element, $i$. To temporally align the received signals from each elements the equation from [2] is used to calculate the required delay $\tau_{i}$. The computed time delay vector added to the received RF signal, $s_{i}(t)$ is known as the aligned RF signal, $v_{i}(t)$ and can be represented by the following equation:

$$
v_{i}(t)=s_{i}\left(t-\tau_{i}\left(x, z, \theta_{n}\right)\right)
$$

Unlike DAS, the aligned signals will go through a process that similar to autocorrelation instead of summation. This will form each image line and is represented by the following equation [6]:

$$
\begin{array}{r}
r_{\text {dmas }}(t)= \\
\sum_{i=1}^{E-1} \sum_{m=i+1}^{E} \operatorname{sgn}\left\{v_{i}(t) v_{m}(t)\right\} \times \sqrt{\left|v_{i}(t) v_{m}(t)\right|}
\end{array}
$$

Multiplying two RF signals with the same frequency content will eventually produce harmonics and DC components. Thus a bandpass filter is applied to $r_{d m a s}(t)$ to extract its second harmonics where finally $r_{f d m a s}(t)$ is obtained.

\section{Compounding}

Table I shows the main parameters used for the compounding process. The maximum steering angles sets within the sector angles, $\left[\theta_{\max }^{\circ}, \theta_{\min }^{\circ}\right] \pm 12$. The frames rates calculated at $30 \mathrm{~mm}$ depth with speed of sound of $1540 \mathrm{~m} / \mathrm{s}$.

TABLE I

COMPOUNDING PARAMETERS

\begin{tabular}{lccccccc}
\hline \hline Properties & \multicolumn{1}{c}{ Values } \\
\hline No. of Compounding, $N$ & 1 & 3 & 5 & 7 & 9 & 13 & 25 \\
Angle Increment, $\Delta \theta$ & 0 & 12 & 6 & 4 & 3 & 2 & 1 \\
Frame Rates, $\|k H z\|$ & 26 & 9 & 5 & 4 & 3 & 2 & 1 \\
\hline \hline
\end{tabular}

\section{Experiments}

In order to validate the proposed methods, experiments are performed on wires and a tissue mimicking phantom (O40GSE, CIRS, Virginia, USA). The wires phantom with 120 $\mu \mathrm{m}$ diameter were imaged starting from $20 \mathrm{~mm}$ to $60 \mathrm{~mm}$ depth with $10 \mathrm{~mm}$ spacing while the hypo-echoic sections of the synthetic phantom were imaged at between 10 to $50 \mathrm{~mm}$ depth. All imaging was performed with a 128 element linear array transducer (L3-8/40EP, Prosonics Co. Ltd., Taiwan). The transducer centre frequency is $4.79 \mathrm{MHz}$ and has $57 \%$ bandwidth at $-6 \mathrm{~dB}$. The transducer's was excited and digitized with a multi purpose imaging system (UARPII, University of Leeds, UK) [7]-[10]. The received signals were sampled at 80 MHz. Complete experiments parameters are given in Table II.

TABLE II

EXPERIMENTS PARAMETERS

\begin{tabular}{lc}
\hline \hline Properties & Values \\
\hline Speed of Sound in Water / CIRS Phantom, m/s & 1482 / 1540 \\
Attenuation in Water / CIRS, dB/MHz/cm & 0.002 / 0.5 \\
No of Elements & 128 \\
Transducer Centre Frequency, MHz & 4.79 \\
Transducer Bandwidth 6 dB, \% & 57 \\
Transducer Element Pitch, mm & 0.3048 \\
Sampling Frequency, Tx / Rx, MHz & 160 / 80 \\
Excitation & 2 cycles Sinusoidal \\
\hline \hline
\end{tabular}

\section{Performance evaluation}

In order to evaluate the final B-mode images qualities formed with DAS and FDMAS beamforming techniques, several key performance indicator have been used. The main lobes resolution of the point target was measured on wire phantom located at $40 \mathrm{~mm}$ depth with Full width half maximum (FWHM), $-6 \mathrm{~dB}$ [11]. While the image CR and CNR of hypoechoic cyst was computed on CIRS phantom located at $15 \mathrm{~mm}$ depth. The CR is used to express the detectability of the object contrast between region of interest (ROI) inside the cyst and its background. While CNR is used the measure the cyst contrast with speckle or noise variation inside and outside of the cyst [6]. High CNR value means cyst can be visualize easily and the acoustic noise standard deviation is small or more uniform. Both CR and CNR equation are given by [12]

$$
\mathrm{CR}(\mathrm{dB})=20 \log _{10}\left(\frac{\mu_{\text {cyst }}}{\mu_{\text {back }}}\right)
$$

$$
\mathrm{CNR}(\mathrm{dB})=20 \log _{10}\left(\frac{\left|\mu_{\text {cyst }}-\mu_{\text {Back }}\right|}{\sqrt{\left(\sigma_{\text {cyst }}{ }^{2}+\sigma_{\text {Back }}{ }^{2}\right)}}\right)
$$

Where $\mu_{\text {cyst }}$ and $\mu_{\text {Back }}$ are means of image intensities inside and outside of the cyst respectively while $\sigma_{c y s t}{ }^{2}$ and $\sigma_{B a c k}{ }^{2}$ are their variances. CR and CNR were calculated on the cysts by creating two different regions with the same dimensions. The first region is inside the cyst while the other region is located outside the cyst at the same depth. This is to ensure that the attenuation caused by the depth does not affect the measurements.

\section{RESUlTS}

Fig. 1 shows an example of the UM operation after the 1st iteration. The process takes place in lateral direction signal of the cyst between $-12 \mathrm{~mm}$ to $-4 \mathrm{~mm}$ as shown in Fig. 4 . The blue color signal represents the errors produced after the subtraction process takes place between the coherent and noncoherent CPWI signals. The resultant positive and negative 


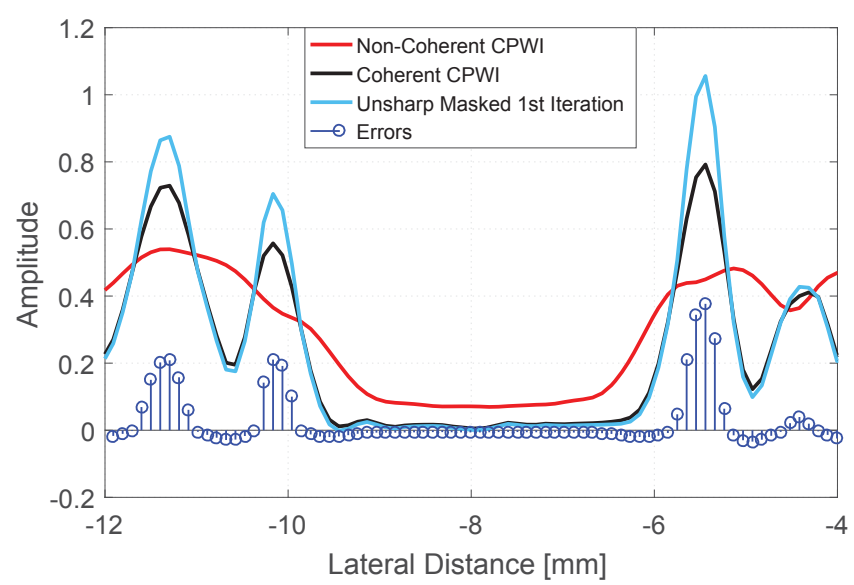

Fig. 1. UM after 1st iteration.

errors signal have been scaled according to equation (2). It is also shown in the figure the UM signals after the 1st iteration.

The B-mode images of point targets compounded with CPWI, $N=13$ angles are shown in Fig. 2. Applying UM on both beamforming techniques shows improvement in noise reduction in the lateral direction. Complete results for lateral resolution (LR) and peak side lobes (PSL) measured on the point targets is presented in graphical form in Fig. 3. Most of the LR improvements takes place within low number of compounding. Increasing the number of compounding with smaller angle increment, $\Delta \theta$, reduced the LR due to more intersection of individual beams in lateral direction [2]. The best LR achieved within $N=3$ to 5 and beyond 7, the main lobes becomes wider as shown in Fig. 3(a).

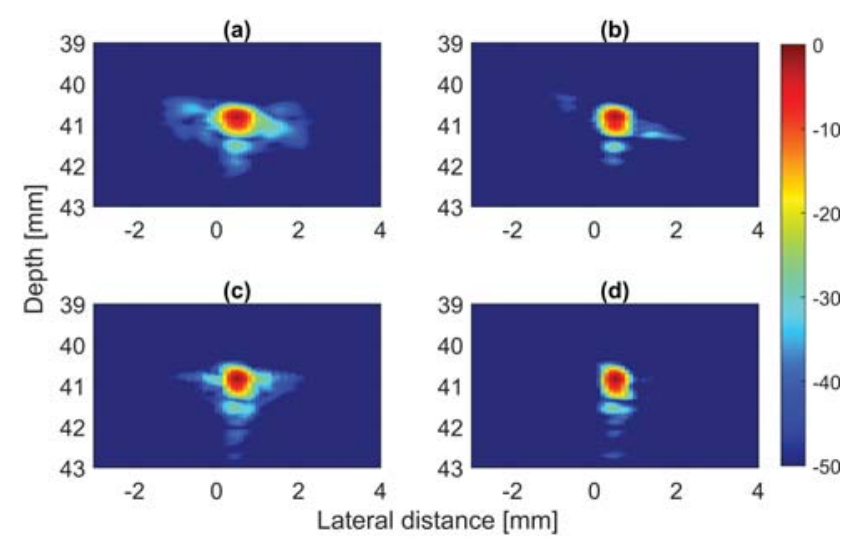

Fig. 2. B-mode image for wire phantom formed with a) DAS, b) UM-DAS, c) FDMAS and d) UM-FDMAS

The limitation imposed by beamforming and compounding techniques on the LR have been overcome by the UM. The LR with DAS, UM-DAS, FDMAS and UM-FDMAS are 0.49 $\mathrm{mm}, 0.40 \mathrm{~mm}, 0.39 \mathrm{~mm}$ and $0.35 \mathrm{~mm}$ respectively with CPWI, $N=13$. This shows that the UM manage to improve the LR by $18.36 \%$ and $10.25 \%$ when applied to DAS and FDMAS (a)

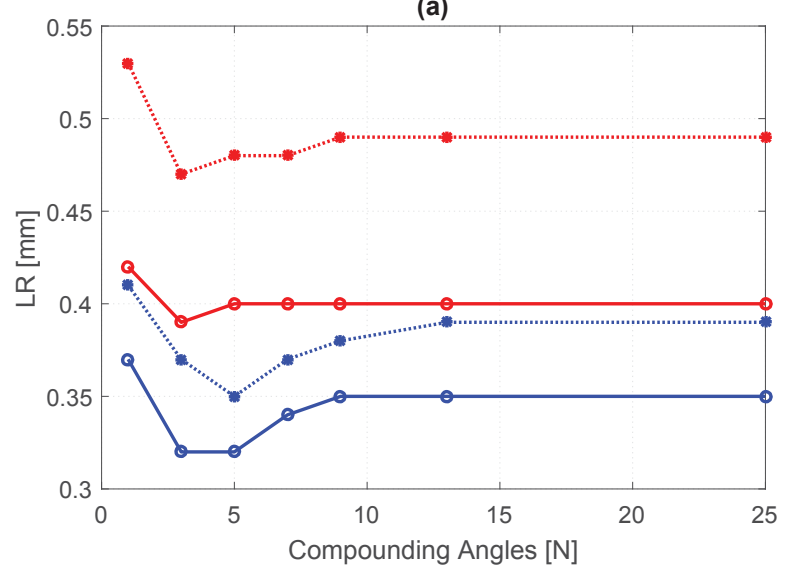

(b)

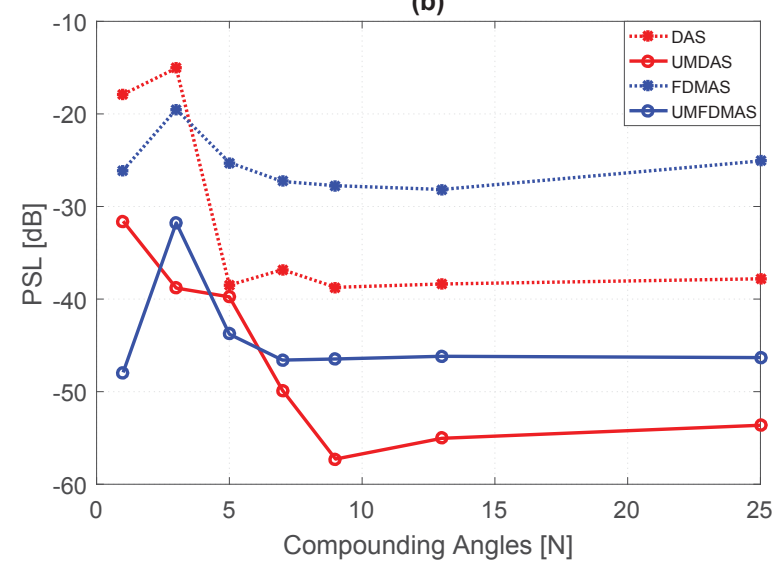

Fig. 3. a) LR and b) PSL results for DAS, UM-DAS, FDMAS and UMFDMAS

respectively. Beyond $N=13$ there are no any changes in LR shown by all the investigated techniques. Overall FDMAS with the UM techniques provide the best LR with all the numbers of compounding.

In general the PSL measured along the lateral direction shows reducing pattern as the number of compounding increases from $N=3$ to $N=9$ and no significant changes takes place beyond $N=13$. Implementing the UM techniques on DAS and FDMAS reduced the PSL by $16.67 \mathrm{~dB}$ and 21.24 $\mathrm{dB}$ respectively when compounded with $N=13$.

The B-mode images of CPWI, $N=13$ for $4.5 \mathrm{~mm}$ and 1.3 $\mathrm{mm}$ diameter CIRS hypoechoic cyst located at $15 \mathrm{~mm}$ depth is shown in Fig. 4. Both of the CR and CNR performance indexes computed on the regions selected inside and outside of the 4.5 $\mathrm{mm}$ diameter cyst. All the CR results as shown in Fig. 5 shows improvements when the number of compounding raised from $N=1$ to $N=25$. Applying UM on DAS and FDMAS enhanced the CR by $10.35 \mathrm{~dB}$ and $7.39 \mathrm{~dB}$ with CPWI, $N=13$. Even though UM-FDMAS performs better than all others from $N=1$ until $N=13$ but at $N=25$, UM-DAS produced better results. Both CR for UM-DAS and UM-FDMAS keep expanding away from DAS and FDMAS correspondingly as the number of compounding increases. 


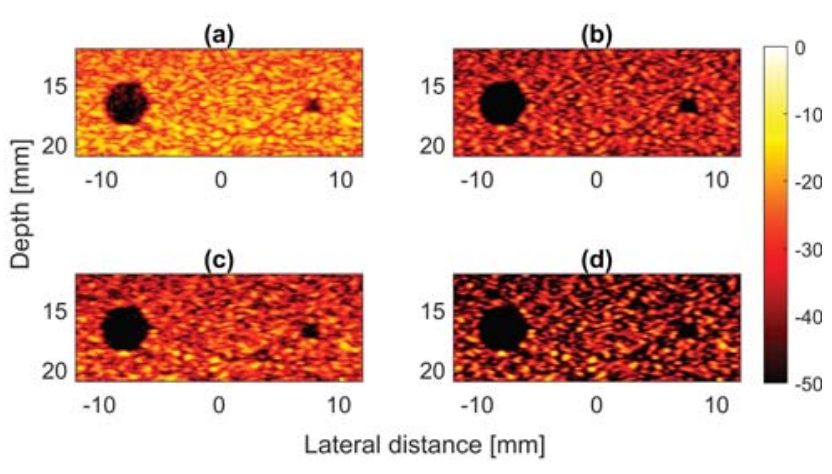

Fig. 4. B-mode image for cysts phantom formed with a) DAS, b) UM-DAS, c) FDMAS and d) UM-FDMAS

Contradicting to all other performance indexes, CNR values tend to decrease when the UM techniques applied to DAS and FDMAS. The CNR for DAS, UM-DAS, FDMAS and UMFDMAS changes by $+0.91 \mathrm{~dB},+0.43 \mathrm{~dB},+0.30 \mathrm{~dB}$ and -1.12 dB from CPWI, $N=1$ to $N=13$. This shows that compounding able to improves the CNR values for all investigated techniques except for UM-FDMAS. With the UM implementation, DAS and FDMAS shows $-4.37 \mathrm{~dB}$ and $-4.29 \mathrm{~dB}$ reduction with CPWI, $N=13$ as shown in Fig. 4. Even though the clutter noise reduction inside the cysts improves the CR but different scenario takes place within the speckle regions. The elimination of the clutter noise makes more prominence to the speckle destructive region. This emphasize the black spots thus leads to low CNR as can be seen on the UM-DAS and UM-FDMAS results as shown in Fig. 4.

\section{CONClusion}

The measured key performance indexes on the B-mode images shows that the new proposed UM techniques able to improve the image contrast and spatial resolution. Both beamforming techniques, DAS and FDMAS shows improvement with UM. The ability of the UM techniques to reduce the clutter noise helps to define the border lines and better visualization the cysts region.

\section{REFERENCES}

[1] A. M. Moubark, S. Harput, D. M. J. Cowell, and S. Freear, "Clutter noise reduction in b-mode image through mapping and clustering signal energy for better cyst classification," in 2016 IEEE International Ultrasonics Symposium (IUS), Sept 2016, pp. 1-4.

[2] Z. Alomari, S. Harput, S. Hyder, and S. Freear, "Selecting the number and values of the cpwi steering angles and the effect of that on imaging quality," in 2014 IEEE International Ultrasonics Symposium, Sept 2014, pp. 1191-1194.

[3] C. H. Seo and J. T. Yen, "Evaluating the robustness of dual apodization with cross-correlation," IEEE Transactions on Ultrasonics, Ferroelectrics, and Frequency Control, vol. 56, no. 2, pp. 291-303, February 2009.

[4] I. K. Holfort, A. Austeng, J. F. Synnevg, S. Holm, F. Gran, and J. A. Jensen, "Adaptive receive and transmit apodization for synthetic aperture ultrasound imaging," in 2009 IEEE International Ultrasonics Symposium, Sept 2009, pp. 1-4.

[5] V. Dutt and J. F. Greenleaf, "Adaptive speckle reduction filter for $\log$ compressed b-scan images," IEEE Transactions on Medical Imaging, vol. 15, no. 6, pp. 802-813, Dec 1996 (a)

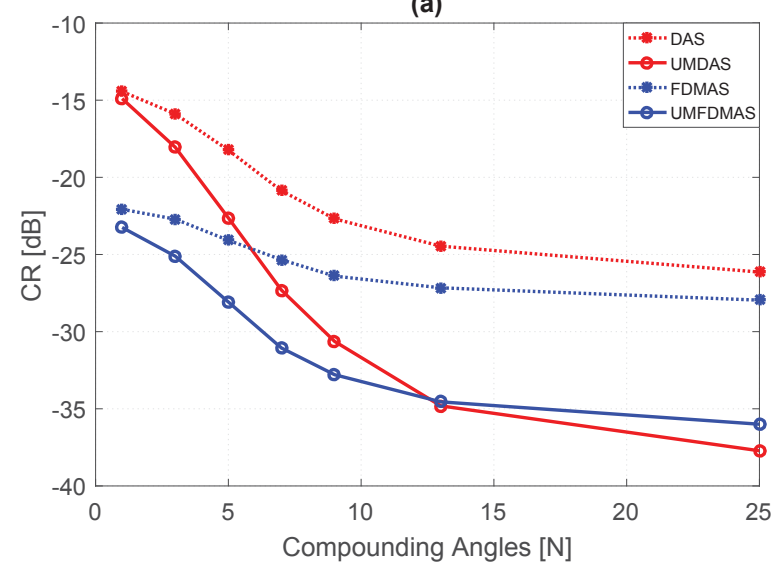

(b)

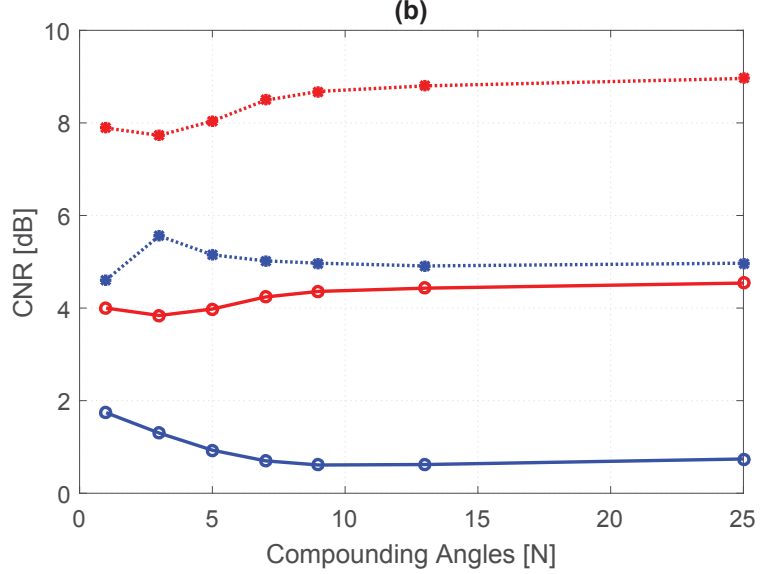

Fig. 5. a) CR and b) CNR for DAS, UM-DAS, FDMAS and UM-FDMAS

[6] G. Matrone, A. S. Savoia, G. Caliano, and G. Magenes, "The delay multiply and sum beamforming algorithm in ultrasound b-mode medical imaging," IEEE Transactions on Medical Imaging, vol. 34, no. 4, pp. 940-949, April 2015.

[7] P. R. Smith, D. M. J. Cowell, and S. Freear, "Width-modulated squarewave pulses for ultrasound applications," IEEE Transactions on Ultrasonics, Ferroelectrics, and Frequency Control, vol. 60, no. 11, pp. 22442256, November 2013.

[8] D. M. J. Cowell, P. R. Smith, and S. Freear, "Phase-inversion-based selective harmonic elimination (pi-she) in multi-level switched-mode tone- and frequency- modulated excitation," IEEE Transactions on Ultrasonics, Ferroelectrics, and Frequency Control, vol. 60, no. 6, pp. 1084-1097, June 2013.

[9] P. R. Smith, D. M. J. Cowell, B. Raiton, C. V. Ky, and S. Freear, "Ultrasound array transmitter architecture with high timing resolution using embedded phase-locked loops," IEEE Transactions on Ultrasonics, Ferroelectrics, and Frequency Control, vol. 59, no. 1, pp. 40-49, January 2012.

[10] S. Harput, M. Arif, J. Mclaughlan, D. M. J. Cowell, and S. Freear, "The effect of amplitude modulation on subharmonic imaging with chirp excitation," IEEE Transactions on Ultrasonics, Ferroelectrics, and Frequency Control, vol. 60, no. 12, pp. 2532-2544, Dec 2013.

[11] S. Harput, J. McLaughlan, D. M. J. Cowell, and S. Freear, "New performance metrics for ultrasound pulse compression systems," in 2014 IEEE International Ultrasonics Symposium, Sept 2014, pp. 440-443.

[12] A. M. Moubark, Z. Alomari, S. Harput, D. M. J. Cowell, and S. Freear, "Enhancement of contrast and resolution of b-mode plane wave imaging (pwi) with non-linear filtered delay multiply and sum (fdmas) beamforming," in 2016 IEEE International Ultrasonics Symposium (IUS), Sept 2016, pp. 1-4. 